\section{BRS: A program for computer-managed point economies}

\section{MICHAEL J, DILLON and FRANS VAN HAAREN Western Michigan University, Kalamazoo, Michigan 49008}

Description. Point or token economies (Kazdin \& Bootzin, 1972) are frequently used to manage complex social systems. Points and tokens are effective, presumably, because they provide immediate feedback on behavior, mediating between current behavior and delayed consequences. After repeated pairing, they may eventually serve as conditioned consequences. For example, in a supervisory system for accomplishing long-range projects (Dillon, Kent, \& Malott, 1980), positive and negative points were assigned for completing or failing to complete research tasks by weekly deadlines.

The present computer program manages the weekly point procedures for this supervisory system. It allows classification of students into four different groups, calculates completion percentages on 11 separate tasks, and provides both weekly and cumulative feedback for the individual student. Thus, the program provides two sources of data: current information on performance to each student, and descriptive statistics to the manager, indicating system performance and permitting evaluation of effectiveness and planning of future changes.

Input. Triannually, the operator enters a 4-month calendar of dates (January-April, May-August, SeptemberDecember).

On a weekly basis, the operator enters students' names, group codes, task scores $(+1$ to +6 for task completion by deadline, -1 to -6 for noncompletion, 0 for task not required), and any messages concerning operation of the supervising system. Each of the 11 tasks can be assigned a differential point value to account for greater difficulty of completion.

Output. The program provides the following output for the system manager: (1) weekly and cumulative totals on number of students, number of required points and percentage of total points completed and not completed for the four groups, and (2) weekly totals on number of students, number of required points, and percentage completed and not completed for each of the 11 tasks, plus the number of extra tasks done.

For the individual student, a feedback form provides the following output: a calendar indicating the current week, the tasks required and completed for that week, cumulative percentage of points completed and not completed for the student and his reference group, and a message space.

Language and Computer. Written in BASIC, the main program requires 105 blocks of storage on the PDP-10 computer (1 block equals 128 5-ASCII-character words). Execution time is about $15 \mathrm{sec}$ for 15 students.

Options. The program provides the following options: (1) Any number of students can be entered; output can be on a daily basis. (2) For the system manager, the program will calculate the percentage of points not completed for groups, separate tasks, and individual students but will omit reporting of the negative points on the feedback form. (We have found that the use of negative points is often considered too aversive by our students.) (3) Easy modification of this program to fit other supervisory point systems is provided by extensive documentation.

Availability. A program listing can be obtained without charge from Richard W. Malott, Department of Psychology, Western Michigan University, Kalamazoo, Michigan 49008.

\section{REFERENCES}

Dil,Lon, M. J., Kent, H. M., \& Malot', R. W. A supervisory system for accomplishing long-range projects: An application to master's thesis research. Journal of Organizational Behavior Management, 1980, 2, 213-227.

Kaz.DIN, A, E., \& Boortaln, R. R. The token economy: An evaluative review. Journal of Applied Behavior Analysis, 1972 , $5,343-372$

(Accepted for publication July 10, 1980.) 